\title{
Operation and Failure of a Hairpin Nucleate Generator on a Bubble Memory Chip
}

\author{
RYO SUZUKI AND FLOYD B. HUMPHREY, FELLOW, IEEE
}

\begin{abstract}
The dynamic operation as well as the failure modes of a hairpin nucleate bubble generator operating at $125 \mathrm{kHz}$ has been studied using a high-speed optical sampling system. The hairpin generator loop consists of a $6.8-\mu \mathrm{m}$ wide $4.2-\mu \mathrm{m}$ gap conductor located under a $20-\mu \mathrm{m}$ period $110^{\circ}$ chevron propagation pattern. In stable operation in the center of the operating range, bubbles are nucleated along the hairpin conductor preserving a $U$ shape that reflects the shape of the hairpin conductor. After nucleation, the gap of the conductor fills with a long wide domain. This long domain then shrinks to a stable position under the propagation elements with a wall velocity of $40 \mathrm{~m} / \mathrm{s}$. All velocities observed are consistent with velocities observed on free bubbles using radial expansion, except for the growth in length after nucleation during the generate pulse, where the domain grows about three times faster than would be expected. This difference is attributed to the inplane component of the generate current field that is very nonuniform through the thickness of the sample. The failure mode for a low or short generate pulse is multibubble generation caused by nonuniform nucleation along the length of the conductors of the hairpin loop. For certain pulse characteristics at this low end of the operating range, a stable U-shaped domain is nucleated only to be cut into two domains at the end of the generate pulse as the drive field reverses the poles on the nearest chevron stack from what it was during the pulse. The failure mode at the upper end of the operating range also results in excess bubbles. Here the mechanism can be identified as the development of a wavy wall as it moves rapidly under the conductor element in the presence of a large in-plane field caused by the generate current. This distortion then increases as the domain shrinks, resulting in nobs that are pinched off to create excess bubbles. The distortion is similar to that seen during the collapse of highly expanded stripes. This failure can be cured by increasing the fall time of the generate pulse to a least $100 \mathrm{~ns}$.
\end{abstract}

\section{INTRODUCTION}

$T$ THE NUCLEATE GENERATOR most commonly used in field access bubble devices to create data bubbles is a hairpin-shaped conductor in a propagation track [1]. The high and localized field from the conductor reverses a region at the surface of the material that then grows into a stable domain. Studies of the temperature dependence of nucleate generator operation has confirmed general features of the model and its dependence on $H_{k}$ [2]. An extensive study of the lower end of the operating range associated the generation of un-

Manuscript received March 17, 1980; revised July 9, 1980. This work was supported by the National Science Foundation Division of Materials Research under Grant DMR 77-24024. A summary of this work was presented at the 1980 INTERMAG Conference, Boston, MA, April 21-24.

R. Suzuki is with the California Institute of Technology, Pasadena, CA 91125, on leave from the Central Research Laboratory, Hitachi Limited, Kokubunji, Tokyo 185, Japan.

F. B. Humphrey was with the California Institute of Technology, Pasadena, CA 91125. He is now with the Electrical Engineering Department, Carnegie-Mellon University, Pittsburgh, PA 15213. wanted bubbles with large stresses introduced during circuit fabrication [3]. Multiple bubble generation is also the failure mechanism at the upper end of the operating range and the sensitivity of this failure to pulse shape has been observed [4] In this paper we report on the detailed mechanism of bubble generation using a hairpin conductor and study the mechanism of failure at both the upper and lower end of the operating range margin that leads to multiple bubbles.

\section{EXPERIMENTAL}

A block diagram of the optical sampling system used in this investigation is shown in Fig. 1. The optical system is similar to that previously used [5] and is described in detail elsewhere [6]. The system is synchronized to the $60-\mathrm{Hz}$ frame rate of the television camera. The trigger unit initiates a sequence in the bubble memory controller (BMC) synchronized with the vertical fly back signal of the video. The controller, which continuously provides a $125 \mathrm{-kHz}$ rotating field, starts a read/ write sequence beginning with a transfer-out operation. Usually, the one address scan mode is used with a word consisting of a single one in a field of many zeros. During the chip operation, the BMC provides a trigger to the laser control unit at the beginning of the cycle of interest. The trigger is incrementally delayed by the delay unit to provide the 10-ns optical sample at a present time during the cycle. This phase (or delay) can be automatically scanned, with a variable increment, providing an optical sample systematically through the entire cycle of interest with each picture from the succeeding BMC sequence. Any question of reproducibility from sequence to sequence can be easily resolved by observing a number of pictures at the same phase. A portion of the laser light is directed onto a fast position-indicator (PIN) photo diode so that the time of the picture can be monitored with other events on the chip using the high-frequency oscilloscope. The image processor adds the frame numbers as well as controller and delay status to the video before the picture is recorded for future processing. For device operation, the device is viewed through the substrate using the reflection (epi-mode) with the mirror necessary for this mode provided by the conductor and the Permalloy film used for hard bubble suppression.

The device studied here is a complete $64-\mathrm{kbit}$ chip with $20-\mu \mathrm{m}$ period propagation elements described in detail elsewhere [7]. The nucleate generator is a hairpin loop design with two $6.8-\mu \mathrm{m}$ wide conductors spaced $4.2 \mu \mathrm{m}$ edge to edge. The end of the $50-\mu \mathrm{m}$ loop is in a $110^{\circ}$ chevron propagation pattern. 


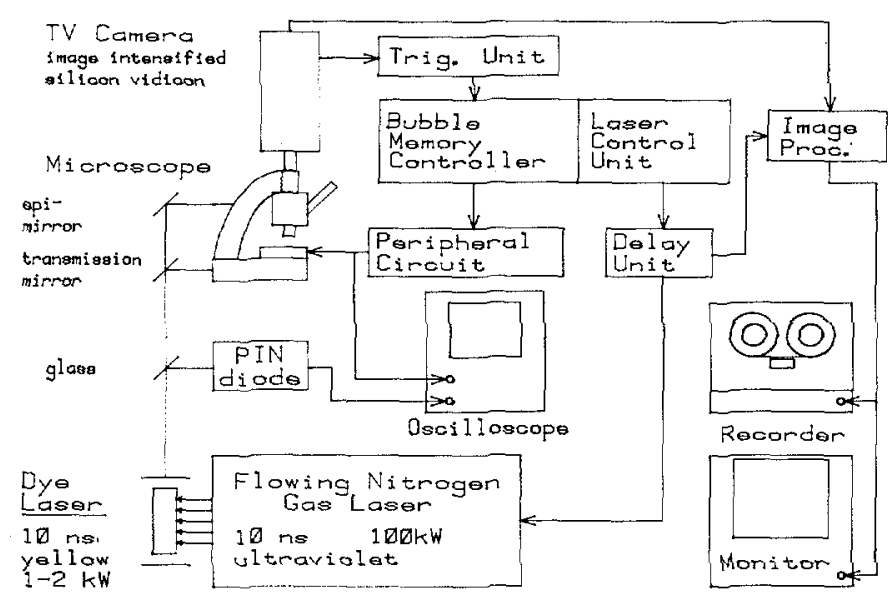

Fig. 1. Optical sampling system.

The conductor at the base of the loop widens to $30 \mu \mathrm{m}$ for $30 \mu \mathrm{m}$, then to $100 \mu \mathrm{m}$, keeping the $4.2-\mu \mathrm{m}$ edge to edge spacing. Hard bubbles are suppressed by a 100- $\AA$ thick Permalloy layer evaporated directly onto garnet [8]. The garnet film has a nominal composition (YSmLuCa) ${ }_{3}(\mathrm{FeGe})_{5} \mathrm{O}_{12}$ with thickness $h=4.8 \mu \mathrm{m}$, demagnetized strip width, $\omega=4.8 \mu \mathrm{m}$, collapse field $H_{c}=118 \mathrm{Oe}$, and uniaxial anisotropy field $H_{k}=$ 1500 Oe. Because of the difference in reflectivity between the Permalloy hard bubble suppression layer and the gold conductor, it is occasionally difficult to see that the domain is continuous through the center of the hairpin loop on the reproduced picture. This ambiguity does not exist on the original so that the sketch associated with each picture is an accurate indication of the domain shape.

All measurements were made with a rotating field of $42 \mathrm{Oe}$ at $125 \mathrm{kHz}$. In the pictures to be seen, the microscope is looking through the substrate with the Permalloy on the far side of the bubble. The rotating field is clockwise, and the bubbles propagate up. Zero phase angle is when the rotating field is along the length of the nucleate loop pointing toward the shorted end. The chip temperature was $50 \pm 5^{\circ} \mathrm{C}$. The bias was changed as indicated through the propagation operating range which was from 110 to $131 \mathrm{Oe}$. A generate pulse was used with characteristics that changed with the experiment. The pulse amplitude was varied from $190 \mathrm{~mA}$, which is the minimum current for generation, to about $1 \mathrm{~A}$. The pulse length was varied from zero to $1 \mu \mathrm{s}$. The rise and fall time was $20 \mathrm{~ns}$, however, the fall time was changed to as long as $600 \mathrm{~ns}$ for some specific experiments. The specific characteristics of the generate pulse will be indicated when discussing the results.

\section{Results AND Discussion}

The generate function of an operating 64-kbit bubble memory chip was investigated by observing the dynamic domain configuration at various times through the generate cycle. A typical sequence is shown in $\mathrm{Fig}$. 2. The time of each picture is indicated in the figure caption. The generate pulse is applied at $t=0.54 \mu \mathrm{s}$. At the very beginning of the pulse, Fig. 2(a), a $U$-shaped domain is nucleated at the end of hairpin loop conductor and under the Permalloy. The Permalloy elements (a)
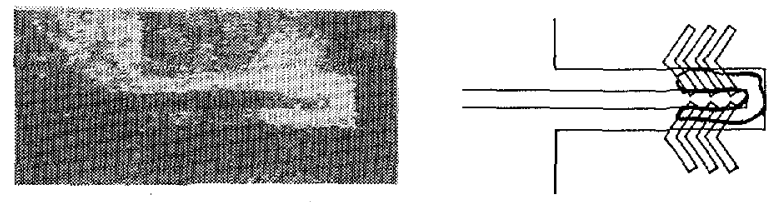

(b)
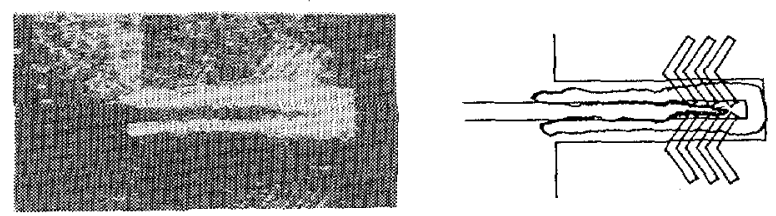

(c)
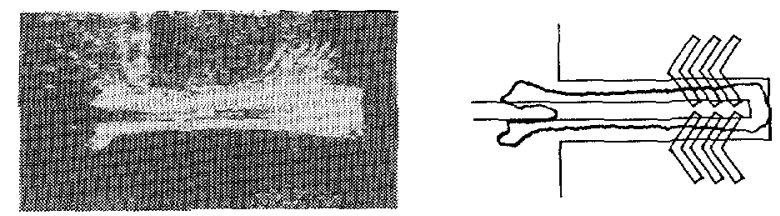

(d)
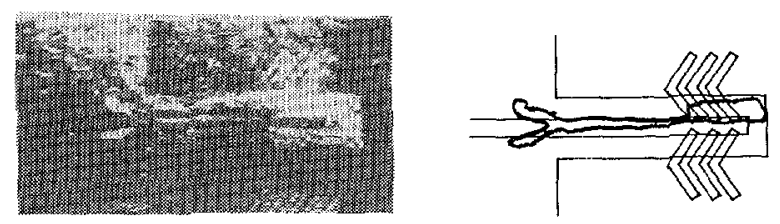

(e)
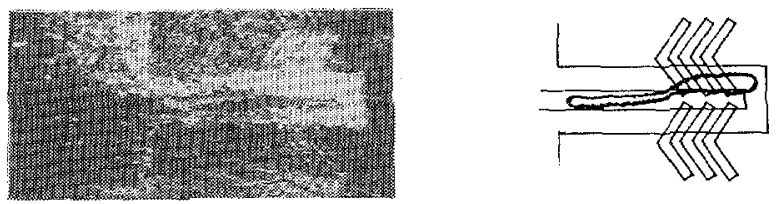

Fig. 2. Dynamic domain configuration after start of 340-ns, 350-mA generate current pulse. (a) At $10 \mathrm{~ns}$. (b) At $70 \mathrm{~ns}$. (c) At $160 \mathrm{~ns}$. (d) At 410 ns. (e) At 1300 ns.

are both magnetized in a direction to aid nucleation by the field from the conductor. The bubble grows rapidly along the conductor, as seen in Fig. 2(b), then inside to fill the gap, as seen in Fig. 2(c). After reaching the wider portion of the conductor, the domain growth speed is reduced as it becomes wider so that the gap is essentially filled by the end of the pulse (not shown). After the pulse termination, the domain starts to shrink in length but more rapidly in width. By $70 \mathrm{~ns}$ after the end of the pulse, the domain width becomes proper for the bias field applied and the domain becomes Y-shaped, as seen in Fig. 2(d). The domain then continues to shrink, changing its shape into an $I$ as seen in Fig. 2(e) before assuming a stable position on the chevron element.

The dynamics of domain generation can be seen more quantitatively in Fig. 3. The position of the growing end of the domain, measured from the shorted end of the loop, is plotted as a function of time. The loop is sketched to the same scale on the right for comparison. The time during which the 400-mA, 20-ns rise and fall time, generate pulse is applied is indicated on the abscissa. The times when the shape is primarily $U, Y$, and $I$ are also indicated. When the domain has two ends extending toward the open end of the generate loop (either U-shaped or Y-shaped), then the position of the longer is indicated by $(+)$ and the shorter by (.). It can be seen that the domain grows rapidly after the generate pulse is applied, filling up the entire loop and 


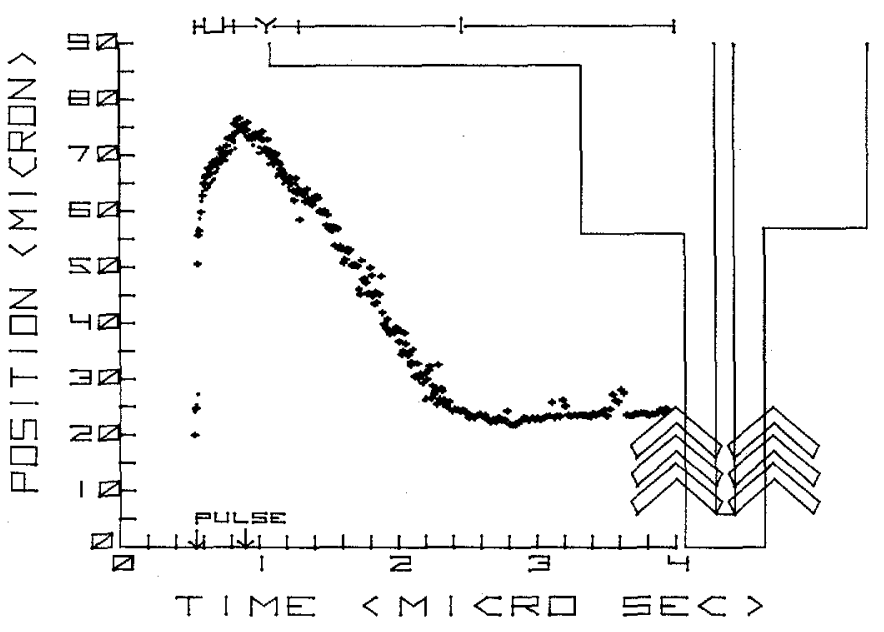

Fig. 3. Observed position of end of generated domain as function of time with application of 340-ns 400-mA generate pulse.

also including a considerable portion of the loop after the conductors have widened. An asymmetry between the two sides is caused by the in-plane field difference on the domain walls under the conductors; the left conductor adds and the right subtracts from the in-plane drive field. During this time the domain is predominately U-shaped, however, very little is left of that shape by the end of the pulse as was seen previously. After the end of the pulse, the domain shrinks, changing shape from the rapidly formed $\mathrm{Y}$ to an I shape before it becomes a stable "bubble" on the chevron about $2 \mu \mathrm{s}$ after the end of the nucleate pulse.

The wall velocity at the tip of the domain at any time during this process is indicated by the slope of the curve seen in Fig. 3 . Because of the rapidly changing shape, however, care must be used when extrapolating from the observed wall position to wall velocity. An extremely high growing velocity of $900 \mathrm{~m} / \mathrm{s}$ is implied during the application of the pulse. This high velocity indicates that domain growth does not occur by usual wall displacement. Magnetization near the surface is reversed along the length of the conductor, not localized at the end, by the pulse current. The depth of this nucleation will depend upon the local anisotropy which may vary along the conductor [3]. Once nucleated, the domain then grows to the other surface by head-on wall displacement. Since neither the magnetic field produced by a pulsed current nor the depth of nucleation is perfectly uniform along the conductor, the time when the reversed domain reaches the other surface varies. Consequently, the domain apparently grows along the conductor with a high speed. It is surprising that the distinct change of slope indicating the end of rotational nucleation is seen at $66 \mu \mathrm{m}$ rather than at $56 \mu \mathrm{m}$ where the widened conductor region begins. When the field from the wide conductors is calculated assuming uniform current distribution, it is too low for rotational nucleation. The observed nucleation in this region must be caused by a very nonuniform current distribution in the wide conductor near the width transition. Finally, it can be seen that the $65-\mu \mathrm{m}$ long nucleated domains grows slightly at $40 \mathrm{~m} / \mathrm{s}$ by wall displacement. After the end of the pulse, the domain shrinks at a fairly uniform velocity,

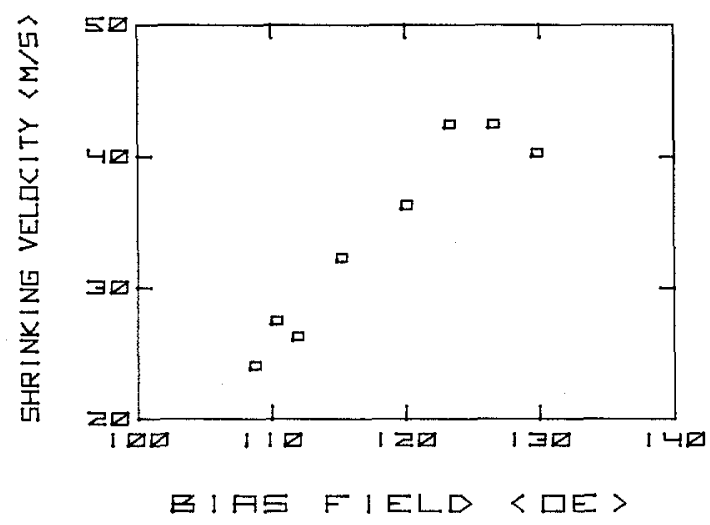

Fig. 4. Shrinking velocity of generated domain as function of bias field.

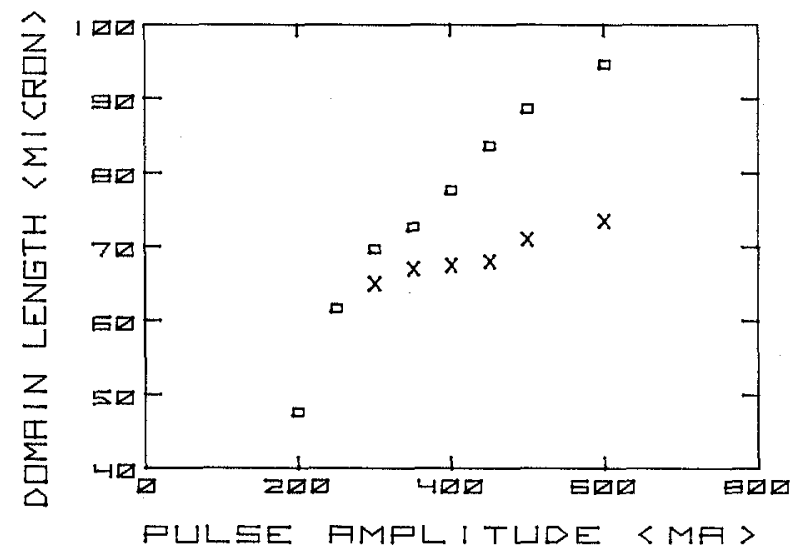

Fig. 5. Maximum length of generated domain as function of magnitude of 340-ns generate current pulse.

a little slower at first $(30 \mathrm{~m} / \mathrm{s})$, then at $42 \mathrm{~m} / \mathrm{s}$. Presumably, this difference in velocity that looks as if it is associated with the conductor width is associated with stress variation during fabrication [3]. The velocity of the shrinking domain tip does not depend on the position of the end nor the length of the domain.

The shrinking velocity depends on the bias field as shown in Fig. 4. Here the average slope after the end of the generate pulse in the region of the narrow conductor is shown as a function of bias field over the entire propagation range. It can be seen that the shrinking velocity increases almost linearly from about $23 \mathrm{~m} / \mathrm{s}$ to $40 \mathrm{~m} / \mathrm{s}$ as the bias field is increased. There is some indication that the shrinking velocity reached saturation at the high bias end of the observation range. This velocity is essentially the same as the "saturation velocity" measured by free bubble radial expansion [9] in the same material. The slope of the linear region and the upper limit of linearity are consistent with the notion that the driving field for the shrinking domain is the difference between the bias and stripe-out field. As expected, the shrinking velocity was independent of domain length, although it was surprising that this independence was also seen very near the Permalloy.

The generator current amplitude established the ultimate size of the domain as shown in Fig. 5. Here the length of the nucleated region $(+)$ as well as the total length of the domain $(\square)$ is shown as a function of current pulse amplitude for the 
340-ns generate current pulse. It can be seen that the nucleated domain is essentially independent of the pulse amplitude as would be expected, since the field under the wide conductors for the highest current used is still less than that needed to nucleate. Once nucleated, the domain moves further into the region of wide conductors as is expected. This increase is more than would be predicted based on the observed free bubble expansion velocity [10] presumably because of the extreme nonuniformity of the field through the thickness of the sample and the increasing in-plane component of the field. This dependence on in-plane field affects the two sides of the domain as expected. Where the drive field adds to the nucleate in-plane field, the growing velocity increases linearly $2 \mathrm{~m} / \mathrm{s} / \mathrm{Oe}$ with increasing drive. Where the field subtracts, it decreases the velocity by $1.5 \mathrm{~m} / \mathrm{s} / \mathrm{Oe}$. Both measurements were made with an $80-\mu \mathrm{m}$ long domain. Finally, when the pulsewidth is increased, the total length increases linearly as would be expected, since the nucleated domain has more time to grow before the end of the pulse.

\section{Failure Modes}

The failure modes for the hairpin generator have been studied by observing the dynamic domain configuration at the generator while it is being operated beyond the margin edge. Fig. 6 is a good example of a failure at the low end of the operation range that generates extra bubbles when the generate pulse is either too short or too low. Fig. 6(a) is the domain structure at the end of a $40-n s$ 350-mA generate pulse with 20 ns rise and fall times. It can be seen that the domain was well nucleated in the vicinity of the Permalloy pattern because of the added fields from the Permalloy. Out from under the Permalloy, however, the domain is narrow and has three bumps. The wider part of the domain is where the wall has been driven to the other surface of the garnet, and the narrow part is where a head-on wall still exists. This structure is similar to that seen previously during a study of stripe chop [10] . The stable domain pattern forms as seen in Fig. 6(b) which was taken 100 ns later. It can be seen that length of stripe away from the Permalloy is being chopped. Finally, in Fig. $6(\mathrm{c})$, it is clear that a stable bubble will form at the ends of the Permalloy elements but that three more bubbles will form in the region away from the Permalloy elements. These extra bubbles are the direct result of the pulse being too short to drive the head-on wall uniformly to the other surface, thereby creating a single stable domain. It is interesting to note that the nonuniformity that chops the stripe into three bubbles is in the structure, since a sequence as shown in Fig. 6 can only be observed when the phenomenon is reproducible. Presumably, this nonuniformity comes from the fabrication process as has been seen previously [3] .

Multiple bubble generation at the lower end of the operating range occurs for pulsewidths slightly greater and slightly less than that seen above but with the same general mechanism. The domain structure shown in Fig. 7(a) is after a 70-ns pulse with the same characteristics as that used in Fig. 6. It can be seen that the pulse is long enough to generate a continuous domain along the conductor where the pulse was too short previously. For a 70 -ns pulse, however, the other side of the (a)
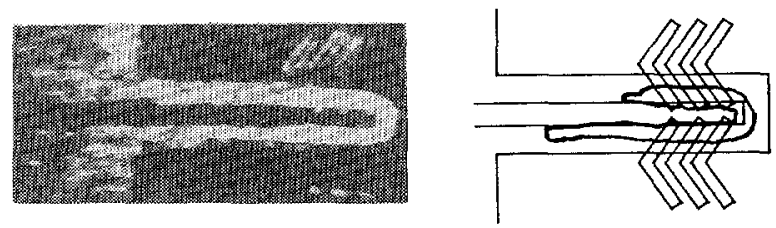

(b)

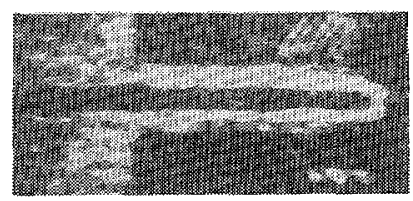

(c)

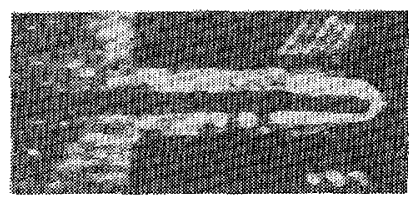

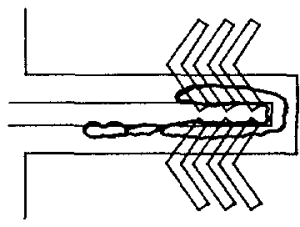

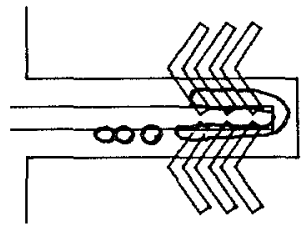

Fig. 6. Pictures of dynamic domain configuration after generate pulse showing failure at low end of operating range. (a) At end. (b) At 100 ns. (c) At $200 \mathrm{~ns}$.

(a)

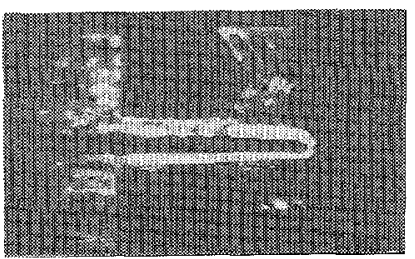

(b)

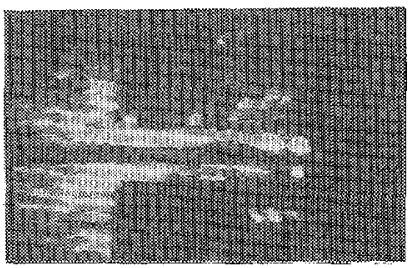

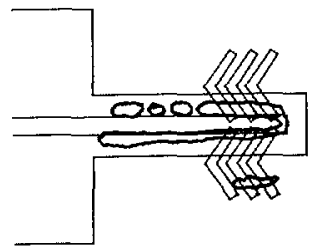

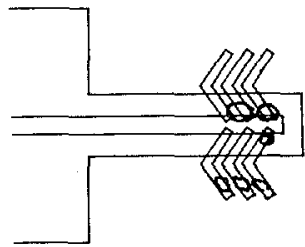

Fig. 7. Pictures showing failure at low end of operating range. (a) At upper edge of multiple bubble range. (b) At lower edge of multiple bubble range.

loop exhibits a similar nonuniformity as was seen previously, resulting in the creation of extra bubbles. Fig. 7(b) shows the domain configuration for a 30-ns pulse with the same characteristics as that used in Fig. 6. Here the time is so short that nucleation and head-on wall displacement only takes place in the field region assisted by Permalloy. The Permalloy chevron ends create a nonuniformity so that multiple bubbles are nucleated. In all cases, the mechanism is similar. Some nonuniformity in the anisotropy of the material, the spacer, the conductor, or the Permalloy causes the initial nucleated domain to be deeper into the material at some places than others. Therefore, the wall reaches the other surface at different times. If the pulse ends before the longest time, multiple domains will be generated.

A second error mechanism observed at the low end of the operating range can be seen when the pulse is low and relatively short. Fig. 8 shows the domain configuration about 100 ns after a) an $80-\mathrm{ns} 350-\mathrm{mA}$ generate pulse and b) an 
(a)

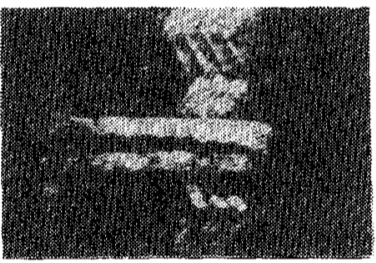

(b)

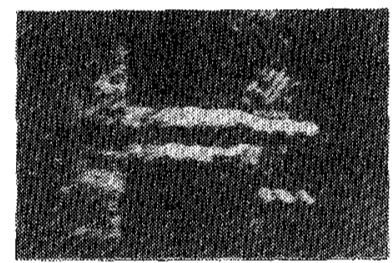

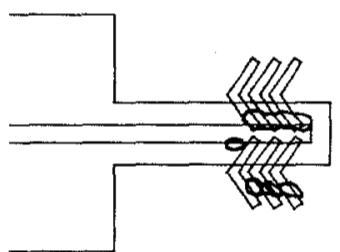

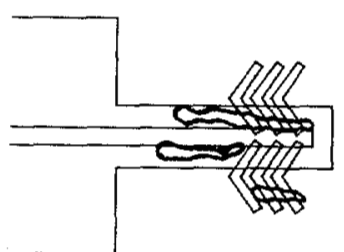

Fig. 8. Pictures taken 100 ns after end of generate pulse showing two examples of stripe chop by Permalloy chevron stack.

80 -ns $380-\mathrm{mA}$ pulse. In both cases, it can be seen that the stripe-like domain was chopped into two stripes causing a multiple bubble failure. By watching this process as a function of time, it can be seen that the chopping occurs after the termination of the generate pulse. This failure can be understood by noting that the generate pulse creates a field at the Permalloy element that aids the instantaneous drive field on the upper chevron stack but is opposite and much larger than the drive field on the lower chevron stack. During the pulse, both chevron stacks encourage the nucleation of the U-shaped domain. Under normal conditions, this U-shaped domain would become one continuous domain as seen in Fig. 2(b) to (c). In this case, however, the generate pulse is terminated, while the U-shaped domain is still present. The Ushaped domain narrows and is chopped by the repulsive poles of the lower chevron stack that is now magnetized properly by the drive field. The stable position for the bubble is further down the chevron into the next cell. In this case, part of the chopped stripe assumes this position adding extra bubbles if none are present as well as extra bubbles external to the track as in Fig. 8(b).

Failure at the upper end of the operating range also results in the generation of excess bubbles. Typical domain shapes are shown in Fig. 9 for various times after a 350-ns 600-mA generate pulse. This pulse is $150 \mathrm{~mA}$ larger than that which will support stable operation. However, this error occurs down to the upper edge of the operating range except it occurs more randomly, so that a pulse well into the failure region was chosen for this example. In this region, the generated domain shown a peculiar shape in addition to the extreme length. At the end of the generate pulse, extreme waviness can be seen in the region of the wide conductor as seen in Fig. 9(a). As the widened stripe relaxes to the equilibrium width for the bias field applied, the protuberances grow as can be seen in Fig. 9(b) taken $40 \mathrm{~ns}$ after the termination of the pulse. Occasionally, the protuberance is so extreme that it pinches off as seen in Fig. 9(c), taken $60 \mathrm{~ns}$ after the end of the pulse. This pinched off domain results in the generation of excess bubbles as seen in Fig. 9(d), taken 100 ns after the end of the pulse. If the bias field is less than free bubble collapse, these extra bubbles hang about the generator and may enter the propagation path leading to errors.

(a)
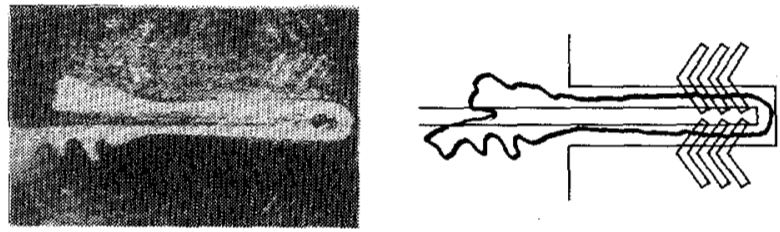

(b)
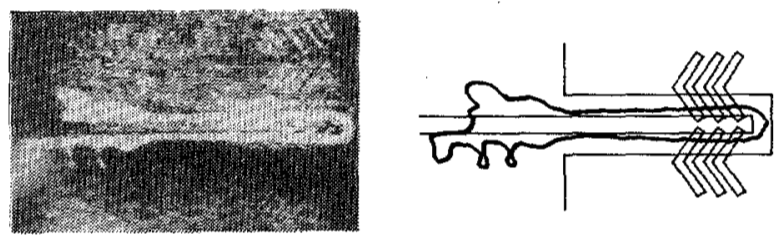

(c)
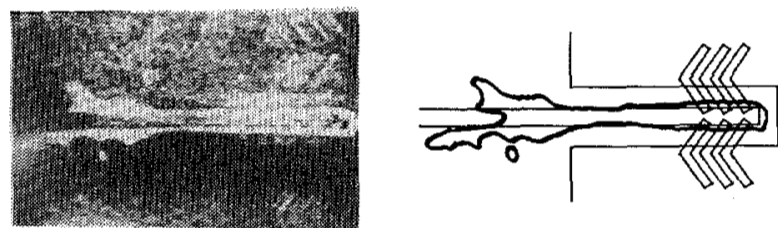

(d)
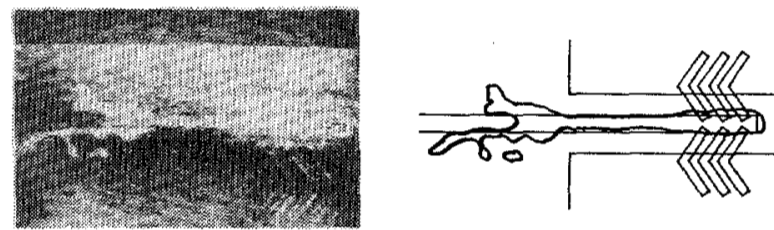

Fig. 9. Pictures taken after 600-mA generate current pulse showing failure at upper end of operating range. (a) At end. (b) At $40 \mathrm{~ns}$. (c) At 60 ns. (d) At $100 \mathrm{~ns}$. (a)

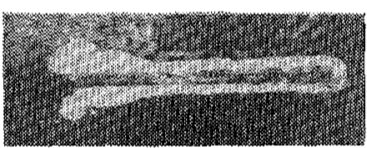

(b)

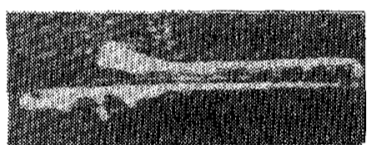

(c)

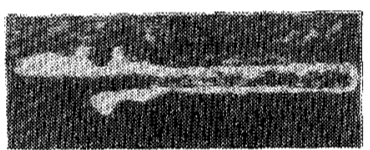

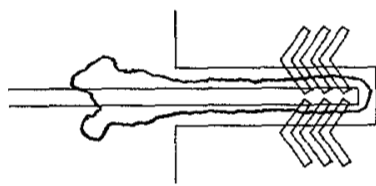
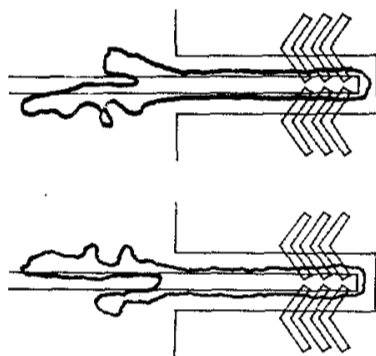

Fig. 10. Dynamics domain configuration at end of $600-\mathrm{mA}$ generate current pulse with instantaneous drive in-plane field drive. (a) Left to right along loop. (b) Down. (c) Up.

The dependence of this failure mechanism on phase can be seen in Fig. 10. These pictures were taken just after the end of a 600 -mA 350-ns generate pulse. The phase for Fig. 10(a) was adjusted to $-8^{\circ}$ so that the center of the generate pulse occurs at $0^{\circ}$ when the drive field is along the hairpin loop. It can be seen that the wall waviness that causes errors does not exist. Fig. $10(\mathrm{~b})$ is with a phase of $90^{\circ}$ which gives essentially the same result as was seen in Fig. 9 where the phase was $25^{\circ}$. This figure should be compared with Fig. 1O(c) where the phase is $270^{\circ}$. It can be seen that the domain structure is nearly the mirror image of Fig. 10(b). The side of the domain that is larger and the side subjected to the waviness is clearly associated with the in-plane drive field. 
(a)

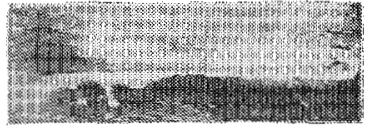

(b)

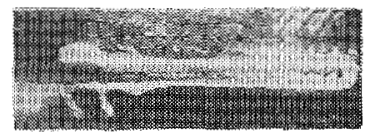

(c)

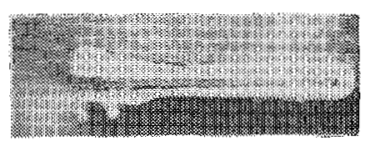

(d)

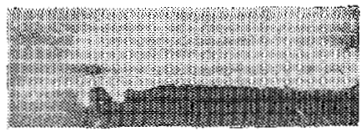

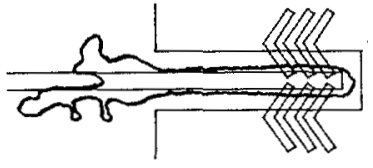
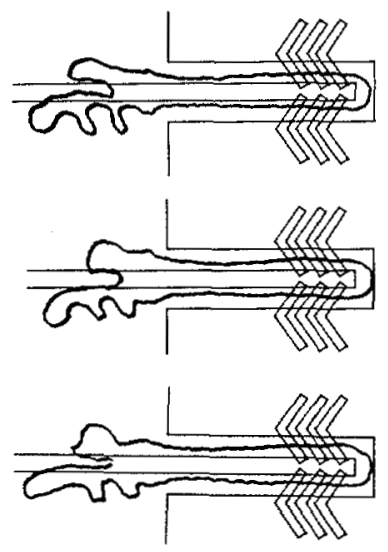

Fig. 11. Dynamic domain pictures see at end of generate current pulse with fall time as follows. (a) $20 \mathrm{~ns}$. (b) $100 \mathrm{~ns}$. (c) $200 \mathrm{~ns}$ (d) $300 \mathrm{~ns}$.

The mechanism for the waviness and distortion that results in excess bubbles is not understood in detail. However, similar and clearly related behavior has been seen before. Expanding stripes develop a wavy structure when the width grows beyond a critical width [12]. This instability has been related to the critical gradient needed to stabilize a straight wall [13]. The distortion observed is very sensitive to the exact relationship between the wall velocity and effective drive. If the wall velocity were truly "saturated," then no distortion can develop even though distortion fields are present. An in-plane field has been shown to affect the "saturation" velocity by making the velocity more sensitive to the drive [10]. Under one stripe conductor, the in-plane component adds to the in-plane drive field, while under the other it subtracts so that there is an average in-plane field difference of twice the drive ( $84 \mathrm{Oe}$ in this case) between the two walls. This asymmetry in field must allow distortion in one wall with higher in-plane field and not in the other. The situation under the conductor is further complicated by the fact that the in-plane field is very nonuniform through the thickness of the film. Once the distortion is established, this case must be similar to the extreme distortion experienced by bubbles (and stripes) when they are at other than their equilibrium size [14]. The distortion fields undoubtedly pinch off the tips after the termination of the pulse as in Fig. 9. Very similar tips are common in stripes after expansion [15] and many times provide the mechanism for stripe chop.

The upper limit of the operating range can be extended by adjusting the phase to the condition shown in Fig. 10(a). Here both walls have equally low in-plane field and hence seem to be out of the in-plane field range that causes distortion. If, however, it is expected to use the same loop for annihilation in the same cycle, there might be restrictions on the phase adjustment. Operating with the fields symmetrically (Fig. 10(a)) only extends the operating range but does not eliminate the problem. A cure for the failure can be implemented by adjusting the fall time of the generate pulse [4]. Fig. 11 shows

the nucleated domain $20 \mathrm{~ns}$ after the end of a $590-\mathrm{mA} 350-\mathrm{ns}$ 20-ns rise time generate pulse the same as that shown in Fig. 10. For Fig. 11(a), the pulse has a fall time equal to the rise time of $20 \mathrm{~ns}$. It can be seen that the distortion fields on collapse will pinch off the tips as was done in Fig. 9. Fig. 11(b) shows the domain configuration when the generate pulse has a 100 -ns fall time. This picture is taken $120 \mathrm{~ns}$ after the pulse started to fall, i.e., $20 \mathrm{~ns}$ after the end of the pulse. It can be seen that the distortion is greatly reduced. Fig. 11(c) and (d) are for a pulse with a fall time of $200 \mathrm{~ns}$ and $300 \mathrm{~ns}$, respectively. It can be seen that the distortion has been so reduced that the multiple bubble failure at the upper margin has been eliminated.

\section{CONCLUSION}

A study of the operation and failure of a hairpin nucleate generator has shown the generator operates much as expected. The domain is nucleated under the conductor reflecting the U-shape of the hairpin loop, and then the center of the loop is filled as the domain grows by wall motion. The velocities are generally consistent with that observed using radial expansion. Failure at the low end of the operating range resulting in excess bubbles was caused by nonuniform nucleation under the conductor, presumably caused by strain introduced during processing. A second mechanism was also identified which involved the chopping of the U-shaped domain by the repulsive poles of the chevron pattern. Failure at the upper end of the operating range which also results in excess bubbles was caused by extreme domain distortion as the oversized domain attempted to shrink to size after the generate pulse. This failure mechanism could be eliminated by using long fall-time generate pulses.

\section{ACKNOWLEDGMENT}

The authors wish to thank the bubble group at Hitachi, Ltd., Japan, particularly Y. Sugita, S. Kurokawa, and K. Yoshida for providing the samples and their encouragement, and $\mathrm{H}$. Aoki for assistance with the electronics. We would also like to thank K. Sato, H. Watanabe, and T. Doi for their arrangement of this work and encouragement.

\section{REFERENCES}

[1] T. J. Nelson, Y. S. Chen, and J. E. Geusic, IEEE Trans. Magn., vol. MAG-9, p. 289, 1973.

[2] I. Danylchuk and A. H. Bobeck, in AIP Conf. Proc., vol. 29, p. $41,1975$.

[3] R. Wolfe and W. A. Johnson, IEEE Trans. Magn., vol. MAG-13, p. $1595,1977$.

[4] S. Matsuyama, R. Konoshita, T. Yanase, I. Otake, and K. Imamma, IEEE Trans. Magn., vol. MAG-15, p. 1715, 1979.

[5] L. Gal and F. B. Humphrey, IEEE Trans. Magn., vol. MAG-15, p. 1113, 1979.

[6] F. B. Humphrey, IEEE Trans. Magn., vol. MAG-11, p. 1679 , 1975.

[7] M. Hiroshima, A. Asano, S. Yoshizawa, N. Saito, and M. Kasai, in Proc. Solid State Device Japan, 7th Conf., 1975, p. 122.

[8] M. Takahashi, H. Nishida, T. Kobayashi, and Y. Sugita, J. Phys. Soc. Japan, vol. 34, p. 1416, 1973.

[9] G. J. Zimmer, L. Gal, K. Vural, and F. B. Humphrey, J. Appl. Phys., vol. 46, p. 4976, 1975.

[10] K. Vural and F. B. Humphrey, J. Appl. Phys., vol. 50, p. 3583, 1979. 
[11] T. J. Gallagher and F. B. Humphrey, Appl. Phys. Lett., vol. 31, p. 235, 1977.

[12] T. M. Morris and A. P. Malozemoff, in AIP Conf. Proc., vol. 18, p. 242,1973 .

[13] F. B. Hagedorn, J. Appl. Phys., vol. 41, p. 1161, 1970.
[14] L. Gal, G. Z. Zimmer, and F. B. Humphrey, Phys. Stat. Sol(a), vol. 30, p. 561,1975 .

[15] G. J. Zimmer, T. M. Morris, and F. B. Humphrey, "Transient bubble and stripe domain configurations in magnetic garnet materials," IEEE Trans. Magn., vol. MAG-10, p. 651, 1974.

\title{
Automated Spatial Filter Station for Bubble Film Characterization
}

\author{
MING-JAU SUN, MEMBER, IEEE
}

\begin{abstract}
A fully automated spatial filter station for bubble film static characterizations is described. The spatial filter is used to measure the diffraction angles of a laser beam transmitted through the bubble film under measurement. The demagnetized domain stripe width is calculated from the first-order diffraction angle for the bubble film under zero bias field, and the saturation magnetization is derived from the bias field which induces a domain periodicity corresponding to a certain value of magnetization in the film. The whole measurement process has been automated, which takes about one minute per wafer, with a precision for domain stripe wid th better than 0.5 percent and that for saturation magnetization 1 pereent.
\end{abstract}

\section{INTRODUCTION}

$\mathrm{T}$ HE PRODUCTION of bubble memory devices requires a fast and reliable station for bubble film characterizations. The conventional method using polarizing microscope to measure domain stripe widths and bubble collapse fields is slow, operator dependent, and not cost effective. The spatial filter technique, first introduced by Henry [1], [2] in 1976, offers a good solution. Magnetic domain patterns act like $180^{\circ}$ phase gratings [3], [4]. Therefore, a linearly polarized laser beam incident on a bubble film will be diffracted. A spatial filter is scanned along the laser beam direction to determine the diffraction angle. The demagnetized domain stripe width is calculated from the first-order diffraction angle for the bubble film under zero bias field, and the saturation magnetization is derived from the bias field which induces a domain periodicity $P$ corresponding to a certain value of magnetization $M$ in the bubble film. For the first time, a fully automated spatial filter station will be reported. The measurement time per wafer is less than $1 \mathrm{~min}$, with a precision for domain stripe width better than \pm 0.5 percent and a precision for saturation magnetization \pm 1 percent.

There is a difference between Henry's measurement processes and this report. In Henry's approach, second-order dif-

Manuscript received April 24, 1980; revised July 21, 1980.

The author is with RD-48, Rockwell International, Anaheim, CA 92803. fraction was used in determining the bias field which induced the domain periodicity corresponding to the value at $M / M_{s}=$ 0.5 , for the reason that the diffraction intensity is a maximum at $M / M_{s}=0.5$ for the second-order diffractions. Actually, the diffraction intensity of the first order is still at least five times larger than that of the second order when $M / M_{s}=0.5$, as shown in Fig. 4. Therefore, first-order diffraction should still be the best choice for determining diffraction angles for bub. ble films under bias field.

The derivations of saturation magnetizations in this experiment are based on the equations derived by Kooy and Enz [5] for the case of straight and parallel domains, but the demagnetized domain patterns of bubble films usually are nonstraight. So it must be proved at least experimentally that the same equations can be used for the actual case. This will be discussed later.

\section{THEORY}

The optical properties of a magnetic phase grating as depicted in Fig. 1 were described by Mezrich and Haskal [3], [4]. Due to Faraday rotation, the polarization of a linearly polarized laser beam is rotated while propagating in a magnetic medium which has a magnetization component in the propagation direction. If the direction of magnetization is reversed, the direction of rotation is also reversed. Therefore, a linearly polarized laser beam incident on a bubble film will have components which are $180^{\circ}$ out of phase at the exit surfaces of the adjacent domains. These components give rise to a farfield diffraction pattern, which consists of a line of light spots if the domain pattern is straight and parallel. This line of diffracted spots is symmetric with the incident laser beam direction and perpendicular to the domain orientation. In actual bubble films, domains are nonstraight and oriented in random directions. In these cases, it can be seen that the diffraction pattern consists of annular rings. The diffraction angle in either case is given by

$$
P \sin \theta=m \lambda
$$

